\title{
Inkjet Printed Capacitive Transducers on Flexible Plastic Substrates with Increase Stability: Ag on PET
}

\author{
Ulrike Altenberend $^{1}$, Francisco Molina-Lopez ${ }^{2}$, Danick Briand ${ }^{2}$, Alexandru Oprea ${ }^{1}$, Nicolae Barsan ${ }^{1}$, \\ Nico F. De Rooij', Udo Weimar ${ }^{1}$ \\ ${ }^{1}$ University of Tübingen, Institute of Physical and Theoretical Chemistry, Auf der Morgenstelle 15, \\ 72076 Tübingen, Germany \\ ${ }^{2}$ Ecole PolytechniqueFédérale de Lausanne, Institute of Microengineering, SAMLAB, Rue Jaquet-Droz \\ 1, P.O. Box 526, CH-2002 Neuchâtel, Switzerland
}

\begin{abstract}
Inkjet printed interdigitated transducers have been produced on flexible substrates and optimized for gas sensing applications by improving the sintering conditions and employing additional passivation procedures, like electroplating with $\mathrm{Ni}$ or whole transducer passivation through Parylene-C coating.

The results of the performed investigations showed that the transducers printed with $\mathrm{Ag}$ ink can be used, as prepared, in capacitive gas sensing structures up to $70 \%$ relative humidity only. Higher humidity levels resulted in irreversible shifts of the nominal capacitance and conductance of the transducers. This drawback could be eliminated by the already addressed passivation procedures or upgraded sintering.
\end{abstract}

The gas sensor structures based on the passivated printed transducers displayed good stability and sensing performance, demonstrating that fully printed and flexible capacitive gas sensors are feasible.

Key words: inkjet printed Ag electrodes, capacitive gas sensor, influence of humidity, plastic substrate

\section{Introduction}

Smart sensing system on foils can be used for goods monitoring, environmental pollution detection and surveillance, smart textiles manufacture, etc. All these application, at least in their mobile versions, ask for low power consumption, low cost materials and production technologies and, if possible, have to be environmentally friendly.

Comparative tests on cheap, commercially available, polymeric foils for electrotechnical use, like poly ethylene therephthalate (PET), poly ethylene naphthalate (PEN) and a polyimide $(\mathrm{PI})$ showed that they are, more or less, suited for the transducer substrates[1].

The low power consumption desiderate can be achieved by using capacitive transducers. According to [2]" $A$ transducer transforms the energy carrying the chemical information about the sample into a useful analytical signal'. In the case of ideal capacitive transducers no principle dissipation comes into play.

Previous investigations on flexible integrated systems for temperature and gas sensing showed good stability of the capacitive transducers from $\mathrm{Pt}$ and $\mathrm{Au}$ patterned by photolithography on PI [3].

In order to reduce the production costs the manufacturing of the structures has to be based on additive technologies only. Therefore the conducting elements of the new sensing systems devised by our groups have been deposited on cheap PET foils by inkjet printing as drop-on-demand technology.

Preliminary results on the inkjet printed silver electrodes, on their stability and on the influence of the electropassivation on the capacitive transducer performance have been carried out and published before [4], [5]. The performed tests, even though encouraging, revealed the need for deeper investigations on the electrode material and its interaction with the substrate and gaseous ambient.

In consequence, the main aim of the reported investigation was the thorough characterization and optimization of the printed transducers. It has been demonstrated that different levels of electrodes stabilization can be achieved by either the optimization of the sintering conditions of the "as printed" Ag-film or by several transducer passivation procedures. 
Humidity, always present in the ambient atmosphere, has been used as main "test gas,, for the study of the electrodes stability.

\section{Experimental}

Inkjet printing as drop-on-demand technique has been used to directly print the electrode material (Ag nanoparticle, ANP DGP 40LT-15C) on a common plastic substrate, namely poly ethylene therephthalate (PET, Melinex ${ }^{\circledR}$ ST506). As it will be shown later on, the transducers, sintered under the conditions recommended by the ink supplier $\left(30 \mathrm{~min}\right.$ at $\left.150^{\circ} \mathrm{C}\right)$ are not stable enough.

For improving the stability of the electrodes two approaches have been followed:

- the optimization of the sintering conditions

- $\quad$ the use of additional passivation steps.

The simplest passivation procedure is the supplementary electroplating of the easy oxidable $\mathrm{Ag}$ electrodes with $\mathrm{Ni}$. Another one is the whole transducer passivation by its coating with Parylene-C monomer under high vacuum conditions. The polymer had to be, subsequently, cross-linked.

As last testing step the passivated transducers have been coated with well known gas sensing materials, such as poly ether urethane (PEUT), in order to evaluate their quality in full sensor structures [3].

The responses of the evaluated samples against gases or vapors have been recorded with suitable instrumentation (HP4263A and HP4285A precision impedance bridges)for different excitation frequencies $(100 \mathrm{~Hz}$ to $10 \mathrm{MHz}$ ). Humidity (delivered in 10\% incremental and decremental pulses and steps from $0 \%$ to $90 \%$ and back) has been considered the main analyte, because, on one

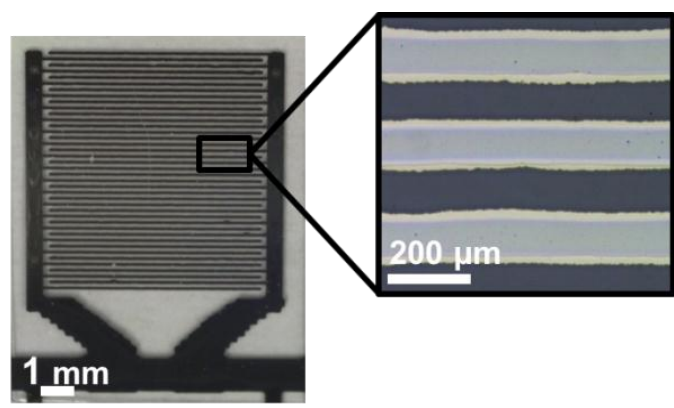

Fig.1. Optical picture of a silver inkjet-printed IDE capacitor, and magnified views of its electrodes. The width of the electrodes and the gap in between them are $95 \pm 3 \mu \mathrm{m}$ and $105 \pm 3 \mu \mathrm{m}$ respectively[4].

hand (as accounted in the introduction), it isaalways present in the ambient at high concentration levels in respect with the other possible target gases, and, on the other hand its action on the substrate and deposited conducting films is very complex (can modify the glassy point of the polymer, favoring thecorrosion of metals, etc.)

\section{Results and Discussion}

Inkjet printed transducers, sintered as proposed by the ink supplier, can be used in capacitive gas sensing applications up to $70 \%$ relative humidity (r.h.). Higher humidity levels result in an irreversible shift of the nominal capacitance and conductance (see Fig.2) of the specimens.

Stability improvements achieved by the optimization of the sintering conditions

The Ag layer stability could be improved by increasing the dwell time in the oven. Instead of sintering for $30 \mathrm{~min}$ at $150^{\circ} \mathrm{C}$, as recommended by the ink supplier, the samples were kept for $2 \mathrm{~h}$ in the oven at the same temperature $\left(150^{\circ} \mathrm{C}\right)$.
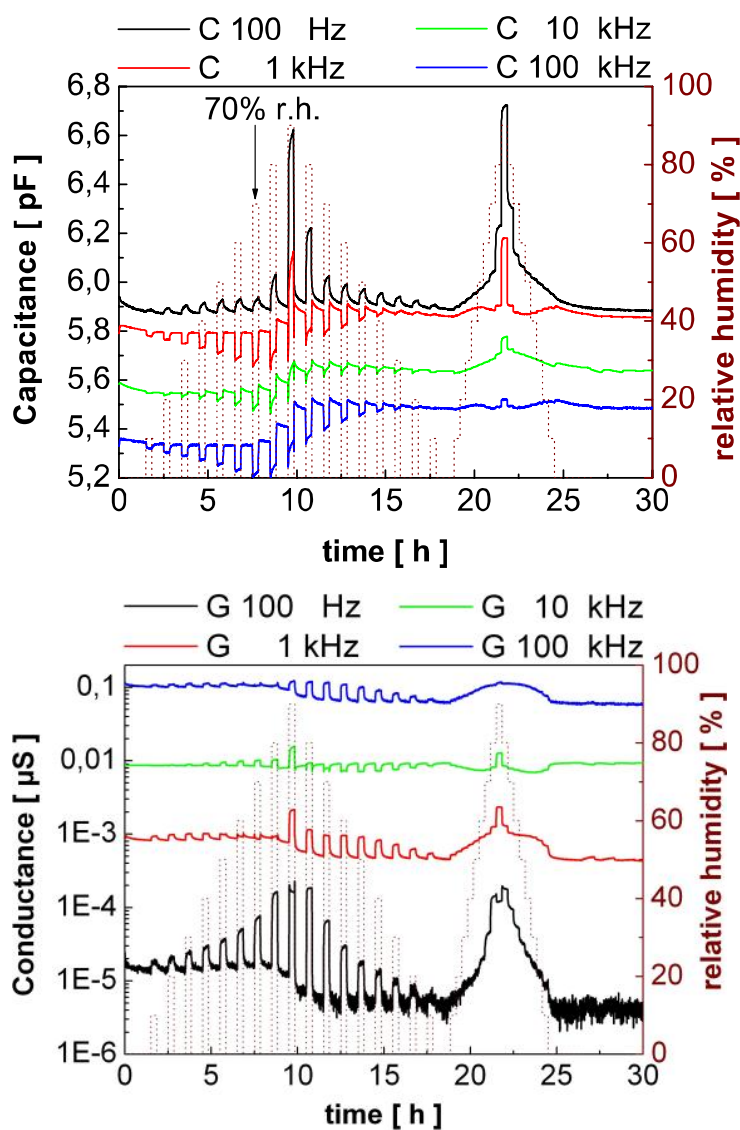

Fig.2 The capacitive (upper panel) and conductive (lower panel) responses of inkjet printed transducers (Ag only) to humidity (dot line). At $70 \%$ r.h. a shift of the nominal capacitance occurs.

After this prolonged sintering step the Ag-film quality significantly increased. Even if exposed to higher humidity (up to $90 \%$ r.h.) the shift encounteredbefore in the nominal capacitance 
and conductance did not occur anymore (see Fig.3).

Although the irreversible shift in the nominal capacitance could be eliminated in this way, a remanent drift of the nominal capacitance and nominal conductancewith the time was still present (see Fig.4). This drift has an exponential behavior indicating a possible stabilization after several weeks of operation.

Comparing the magnitude of drift in the nominal capacitance displayed in Fig.4 with the substrates sensitivity $S$ to humidity (see Table 1 , equation 1), it is evident that there is

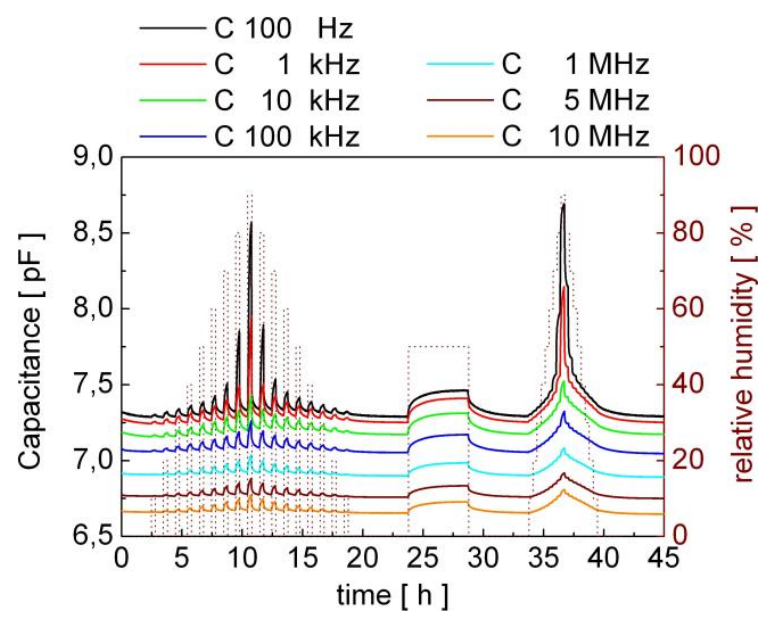

Fig.3 Response of inkjet printed transducers ( $\mathrm{Ag}$ only) to humidity (dot line). The electrodes of the samples undergone improved sintering procedure. Nominal capacity value remains unchanged up to more than $90 \%$ r.h.

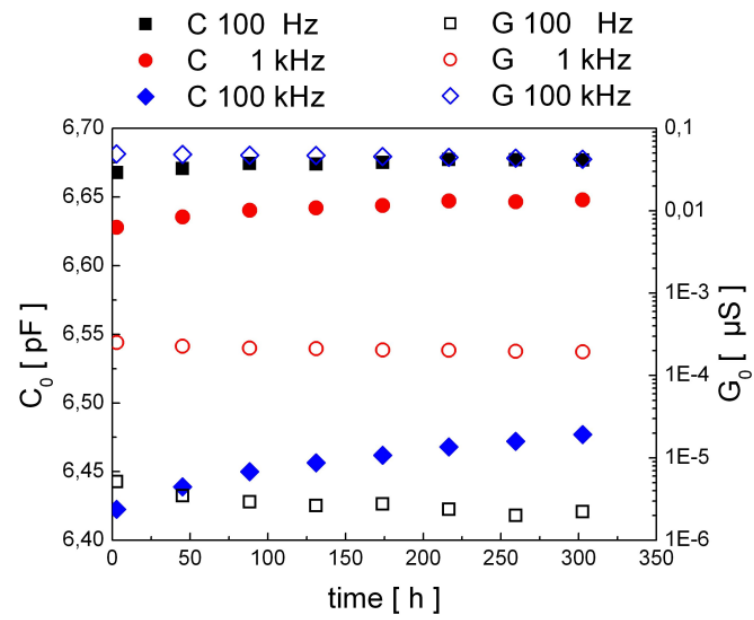

Fig.4 Drift of the nominal Capacitance $C_{0}$ (closed symbols) and nominal conductance Golopen symbols) for the frequencies $100 \mathrm{~Hz}, 1 \mathrm{kHz}$ and $100 \mathrm{kHz}$. The experimental points are only graphicexamples; the measurement was continuous.

still need for further improvement in the sintering conditions.
$S=\frac{d C}{d c}$

Table 1 Frequency dependent sensitivitys ( $\Delta C /$ r.h.) obtained in range of constant change in sensor response with change in analyte concentration.

\begin{tabular}{|c|c|}
\hline Frequency & $\mathrm{S}$ \\
& {$\left[10^{-3} \mathrm{pF} / \%\right.$ r.h. $]$} \\
\hline $100 \mathrm{~Hz}$ & 3,15 \\
\hline $1 \mathrm{kHz}$ & 2,92 \\
\hline $100 \mathrm{kHz}$ & 2,03 \\
\hline
\end{tabular}

Stability improvements achieved by passivation procedures.

The second way approach for electrodes stabilization was the passivation or whole transducer passivation, as already partially reported in [4].

\section{a. Whole transducer passivation}

The performed evaluation under humidity exposure showed that whole transducer passivation with Parylene-C stabilizes the transducer impedance for ambient humidityup to $90 \%$ r.h. but increases the response and recovery times of the transducers (see Fig. 5).

\section{b. Ag electrode passivation with $\mathrm{Ni}$}

Electrodes passivation with $\mathrm{Ni}$ stabilizes both the nominal capacitance and the transducers response (see Fig.6).

The transducer responses towards $50 \%$ relative humidity recorded over a period of more than 4 months display good signals reproducibility which has to be ascribed to increased electrodes stability (see Fig.7).

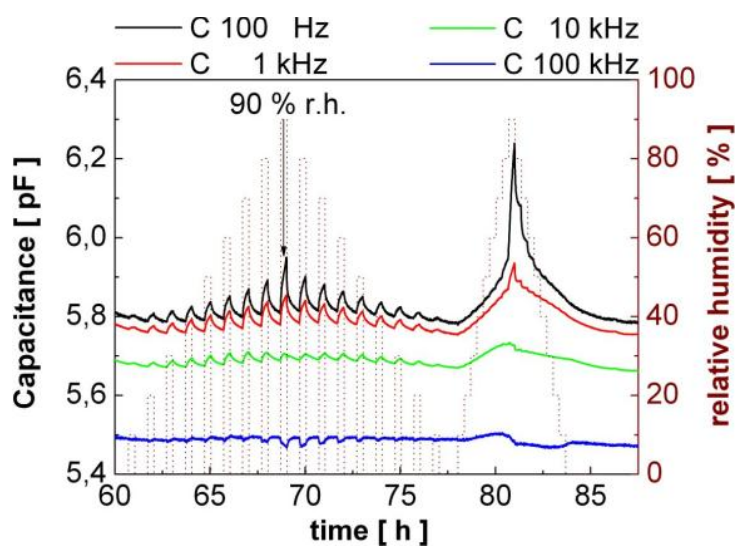

Fig. 5 The capacitive response towards humidity of the transducerspassivated by Parylene $C$. 


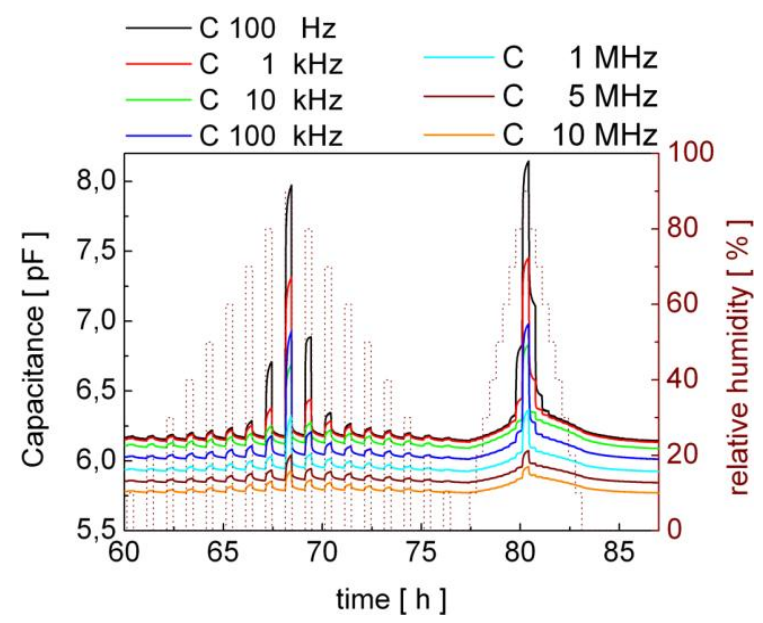

integrated on plastic foils. It makes therefore sense to evaluate the stability of the responses provided by a standard sensing layer which was deposited on such transducers. For instance we address the case of spray coated PEUT. The spray coating (the large area "old brother" of the ink jet printing) has been chosen for its effectiveness on larger areas / low resolution films, as the sensing ones are.

The PEUT example is illustrated in Fig.7 upper curve. The displayed differential response of the capacitive sensor (PEUT/Ni/Ag/PET) has been obtained by subtracting the response of bare $\mathrm{Ni} / \mathrm{Ag} / \mathrm{PET}$ transducer from the total response of the sensor (PEUT/Ni/Ag/PET) [3].

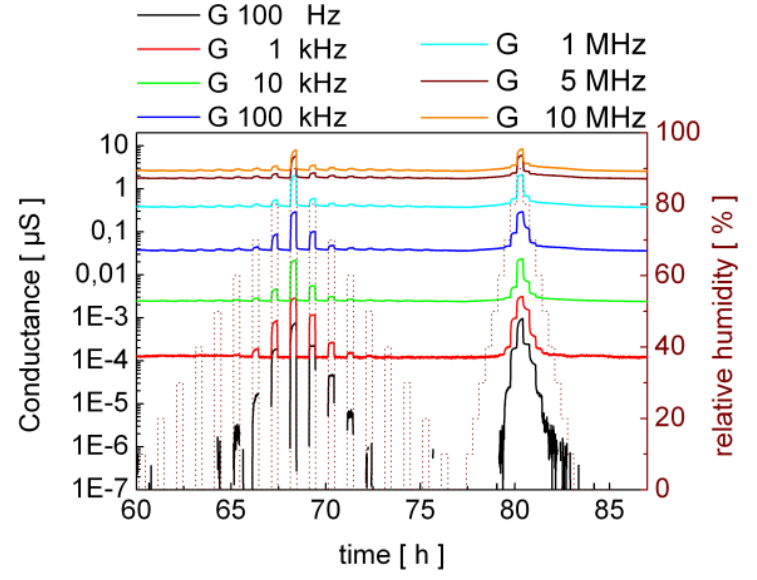

$C_{\text {Difference }}=C_{\text {PEUT }+ \text { substrate }}-C_{\text {substrate }}$

\section{Conclusion}

Capacitive transducersexclusively based on $\mathrm{Ag}$ inkjet printing on PET foils have been manufactured and their stability extensively investigated. The evaluations performed over more than four month demonstrated good device functionality and stability. The upgraded sintering procedures or the use of additional pasivation layers enabled the increase of the humidity operation range from $70 \%$ rh. to $90 \%$ r.h. Whole research results demonstrate that fully printed and flexible capacitive transducers

Fig.6 The capacitive (upper panel) and conductive (lower panel) responsestowards humidity of the transducerspassivated by electrodeposition (Ni, $1 \mu \mathrm{m})$.

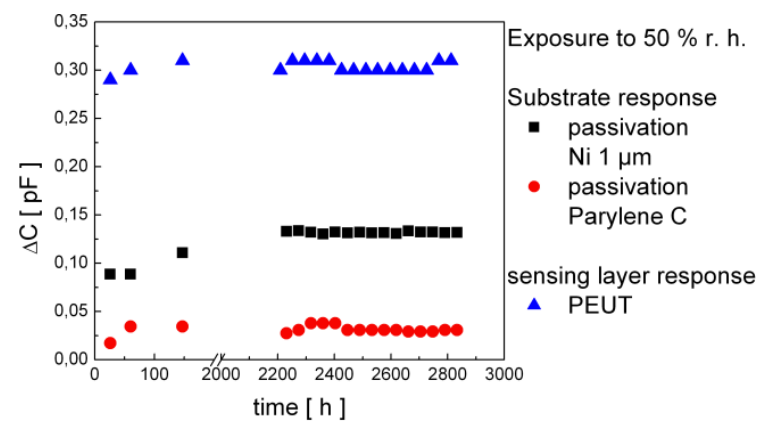
for gas sensors are feasible.

\section{References}

[1] U. Altenberend et al.,in 4th International Symposium on Flexible Organic Electronics (ISFOE-11), (2011).

[2] A. Hulanicki, et al., Pure and Applied Chemistry, 63,9,1247-1250(1991); doi:10.1351/pac199163091247.

[3] A. Oprea, et al., Sensors and Actuators, B: Chemical, 140, 227-232, (2009); doi:10.1016/j.snb.2009.04.019.

Fig.7.Middle term (more than 4 month) stability of differently passivated test specimens. Middle and lower curve describepassivatedtransducers alone(with $\mathrm{Ni}$ and respectively Parylene $\mathrm{C}$ ). The upper curve represents the differential response of a PEUT/Ni/Ag/PET structure. It has been obtained by subtracting the response of bare Ni/Ag/PET transducer from the response of the same transducer coated with PEUT (PEUT/Ni/Ag/PET). Repetitive (at the beginning) and continuous (at the end) sample exposures to pulses of $50 \%$ r.h.

Transducer potential for application in gas sensing systems

The investigated interdigitated transducers have been devised for capacitive gas sensors 